\title{
A PAGE OF MATHEMATICAL AUTOBIOGRAPHY
}

\author{
BY SOLOMON LEFSCHETZ
}

\section{INTRODUCTION}

As my natural taste has always been to look forward rather than backward this is a task which I did not care to undertake. Now, however, I feel most grateful to my friend Mauricio Peixoto for having coaxed me into accepting it. For it has provided me with my first opportunity to cast an objective glance at my early mathematical work, my algebro-geometric phase. As I see it at last it was my lot to plant the harpoon of algebraic topology into the body of the whale of algebraic geometry. But I must not push the metaphor too far.

The time which I mean to cover runs from 1911 to 1924, from my doctorate to my research on fixed points. At the time I was on the faculties of the Universities of Nebraska (two years) and Kansas (eleven years). As was the case for almost all our scientists of that day my mathematical isolation was complete. This circumstance was most valuable in that it enabled me to develop my ideas in complete mathematical calm. Thus I made use most uncritically of early topology à la Poincaré, and even of my own later developments. Fortunately someone at the Académie des Sciences (I always suspected Emile Picard) seems to have discerned "the harpoon for the whale" with pleasant enough consequences for me.

To close personal recollections, let me tell you what made me turn with all possible vigor to topology. From the $\rho_{0}$ formula of Picard, applied to a hyperelliptic surface $\Phi$ (topologically the product of 4 circles) I had come to believe that the second Betti number $R_{2}(\Phi)=5$, whereas clearly $R_{2}(\Phi)=6$. What was wrong? After considerable time it dawned upon me that Picard only dealt with finite 2-cycles, the only useful cycles for calculating periods of certain double integrals. Missing link? The cycle at infinity, that is the plane section of the surface at infinity. This drew my attention to cycles carried by an algebraic curve, that is to algebraic cycles, and $\cdots$ the harpoon was in!

My general plan is to present the first concepts of algebraic geometry, then follow up with the early algebraic topology of Poincaré plus some of my own results on intersections of cycles. I will then discuss the topology of an algebraic surface. The next step will be a

An address delivered at Brown University on April 14, 1967. Submitted by invitation of the editors; received by the editors September 7, 1967. 
summary presentation of the analytical contributions of Picard, Severi and Poincaré leading to my work, application of topology to complex algebraic geometry concluding with a rapid consideration of the effect on the theory of abelian varieties.

This is not however a cold recital of results achieved duly modernized. To do this would be to lose the "autobiographical flavor" of my tale. I have therefore endeavored to place myself back in time to the period described and to describe everything as if I were telling it a half century ago. From the point of view of rigor there is no real loss. Analy tically the story is fairly satisfactory and to make it so in the topology all that is needed is to accept the results amply described in my Colloquium Lectures [10].

To place the story into focus I must say something about what we knew and accepted in days gone by. That is I must describe our early background.

In its early phase (Abel, Riemann, Weierstrass), algebraic geometry was just a chapter in analytic function theory. The later development in this direction will be fully described in the following chapters. A new current appeared however (1870) under the powerful influence of Max Noether who really put "geometry" and more "birational geometry" in to algebraic geometry. In the classical mémoire of BrillNoether (Math. Ann., 1874), the foundations of "geometry on an algebraic curve" were laid down centered upon the study of linear series cut out by linear systems of curves upon a fixed curve $f(x, y)=0$. This produced birational invariance (for example of the genus $p$ ) by essentially algebraic methods.

The next step in the same direction was taken by Castelnuovo (1892) and Enriques (1893). They applied analogous methods to the creation of an entirely new theory of algebraic surfaces. Their basic instrument was the study of linear systems of curves on a surface. Many new birationally invariant properties were discovered and an entirely new and beautiful chapter of geometry was opened. In 1902 the Castelnuovo-Enriques team was enriched by the brilliant personality of Severi. More than his associates he was interested in the contacts with the analytic theory developed since 1882 by Emile Picard. The most important contribution of Severi, his theory of the base (see \$12) was in fact obtained by utilizing the Picard number $\rho$ (see $\$ 11)$.

The theory of the great Italian geometers was essentially, like Noether's, of algebraic nature. Curiously enough this holds in good part regarding the work of Picard. This was natural since in his time Poincaré's creation of algebraic topology was in its infancy. 
Indeed when I arrived on the scene (1915) it was hardly further along.

About 1923 I turned my attention to "fixed points" which took me away from algebraic geometry and into the more rarefied air of topology. I cannot therefore refer even remotely to more recent doings in algebraic geometry. I cannot refrain, however, from mention of the following noteworthy activities:

I. The very significant work of W. V. D. Hodge. I refer more particularly to his remarkable proof that an $n$-form of $V^{q}$ which is of the first kind cannot have all periods zero (see Hodge [13]).

II. The systematic algebraic attack on algebraic geometry by Oscar Zariski and his school, and beyond that of André Weil and Grothendieck. I do feel however that while we wrote algebraic GEOMETRY they make it ALGEBRAIC geometry with all that it implies.

References. For a considerable time my major reference was the Picard-Simart treatise [2]. In general however except for the writings of Poincaré on topology my Borel series monograph [9] is a central reference. The best all around reference not only to the topics of this report but to closely related material is the excellent Ergebnisse monograph of Zariski [11]. Its bibliography is so comprehensive that I have found it unnecessary to provide an extensive one of my own.

\section{TABLE OF CONTENTS}

\section{GENERAL REMARKS ON ALGEBRAIC VARIETIES}

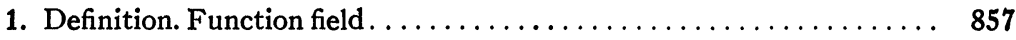

2. Differentials .............................. 858

3. Differentials on curves......................... 858

II. TOPOLOGY

4. Results of Poincaré. .............................. 860

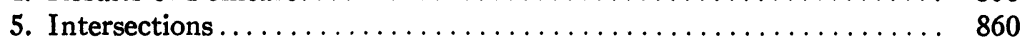

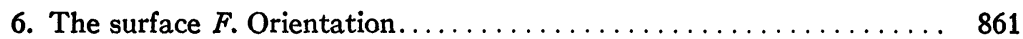

7. Certain properties of the surface $F$. Its characteristic .......... 862

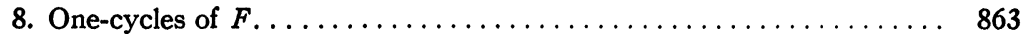

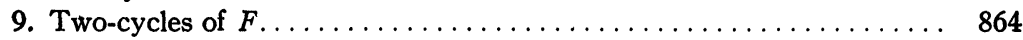

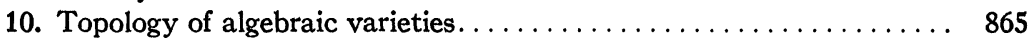

III. ANALYSIS WITH LITTLE TOPOLOGY

11. Emile Picard and differentials on a surface................ 866

12. Severi and the theory of the base $\ldots \ldots \ldots \ldots \ldots \ldots \ldots \ldots \ldots \ldots, 867$

13. Poincare and normal functions. . . . . . . . . . . . . . . . . . 869

IV. ANALYSIS WITH TOPOLOGY

14. On the Betti number $R_{1} \ldots \ldots \ldots \ldots \ldots \ldots \ldots \ldots \ldots \ldots, \ldots 72$

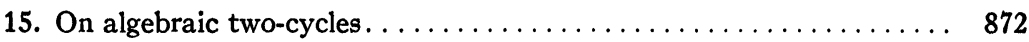

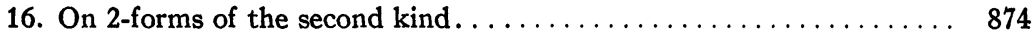

17. Absolute and relative birational invariance.............. 875

18. Application to abelian varieties..................... 876 


\section{General Remarks on Algebraic Varieties}

1. Definition. Function field. It was the general implicit or explicit understanding among algebraic geometers of my day that an algebraic $n$-variety $V^{n}$ ( $n$ dimensional variety) is the partial or complete irreducible intersection of several complex polynomials or "hypersurfaces" of a projective space $S^{n+k}$, in which $V^{n}$ had no singularities (it was homogeneous). Thus $V^{n}$ was a compact real $2 n$-manifold $M^{2 n}$ (complex dimension $n$ ). It could therefore be considered as its own Riemann manifold as I shall do throughout.

For convenience in analytical operations one customarily represents $V^{n}$ by a general projection in cartesian $S^{n+1}$

$$
F\left(x_{1}, x_{2}, \cdots, x_{n}, y\right)=0,
$$

where $F$ is an irreducible complex polynomial of degree $m$. In this representation, the variety, now called $F$, occupies no special position relative to the axes. ${ }^{1}$ As a consequence (1.1) possesses the simplest singularities. For a curve they consist of double points with distinct tangents, for a surface: double curve with generally distinct tangent planes along this curve.

Incidentally, the recent brilliant reduction of singularities by Hironaka [12] has shown that the varieties as just described are really entirely general.

Returning to our $V^{n}$ the study of its topology will lean heavily upon the properties of the pencil of hypersurfaces $\left\{H_{\nu}\right\}$ cut out by the hyperplanes $y=$ const. The particular element of the pencil cut out by $y=c$ is written $H_{c}$. As my discourse will be mostly on surfaces I will only describe (later) certain pecularities for varieties.

Function field. Let the complex rational functions $R\left(x_{1}, \cdots, x_{n}, y\right)$ be identified $\bmod F$. As a consequence they constitute an algebraic extension of the complex field $K$ written $K(F)$, called the function field of $F$.

Let $F^{*}$ be the nonsingular predecessor of $F$ in $S^{n+k}$ and let $\left(u_{1}, \cdots, u_{n+k}\right)$ be cartesian coordinates for $S^{n+k}$. On $F^{*}$ they determine elements $\xi_{h}, h \leqq n+k$ of $K(F)$. The system

$$
u_{h}=\xi_{h}, \quad h \leqq n+k
$$

is a parametric representation of $F^{*} . F^{*}$ is a model of $K(F)$.

Any two models $F_{1}^{*}, F_{2}^{*}$ are birationally equivalent: birationally transformable into one another. The properties that will mainly

1 That is, $F$ has only those singularities which arise from a general projection on $S^{n+1}$ of a nonsingular $V^{n} C S^{n+k}$. 
interest us are those possessing a certain degree of birational invariance (details in \$17).

Terminology. Since only algebraic curves, surfaces, varieties will be dealt with, I drop the mention "algebraic" and merely say curve, . . . .

The symbol $\mho^{n}$ represents a (usually complex) $n$ dimensional vector space.

2. Differential forms. Let $\alpha, \beta, \cdots$, denote elements of the function field $K(F)$. I shall refer to various differentials: zero, one, two, ... forms $\omega^{0}, \omega^{1}, \omega^{2}, \cdots$, in the sense of Elie Cartan of type

$$
\omega^{k}=\sum \alpha_{i_{1}}, \cdots, i_{k} d \alpha_{i_{1}} \cdots d \alpha_{i_{k}},
$$

every $\alpha$ in $K(F)$, as zero, one, two, $\cdots$, forms. They are calculated by the rules of calculus, remembering that the $d \alpha_{j}$ are skew-symmetric, that is $d \beta d \alpha=-d \alpha d \beta$.

Note that $d \omega^{k}$ is an $\omega^{k+1}$ called exact and that if $d \omega^{k}=0$ one says that $\omega^{k}$ is closed.

Special terms are: $\omega^{k}$ is of the first kind when it is holomorphic everywhere on $F$; of the second kind when it is holomorphic at any point of $F$ mod some $d \alpha$; of the third kind if neither of the first nor of the second kind.

The evaluation of the number of kinds one or two constitutes one of the main problems to be discussed.

3. Differential forms on a curve. Let the curve be

$$
f(x, y)=0
$$

and let $m$ be its degree. We refer to it as "the curve $f$." Under our convention, $f$ has no other singularities than double points with distinct tangents and is identified in a well-known sense with its Riemann surface. Its one-forms are said to be abelian. An adjoint to $f$ is a polynomial $\phi_{n}(x, y)$ ( $n$ is its degree) vanishing at all double points.

The following are classical properties:

One-forms of the first kind. They are all reducible to the type

$$
\frac{\phi_{m-3} d x}{f_{y}^{\prime}}
$$

They form a $\mho^{p}$, where $2 p=R_{1}$, the first Betti number of the Riemann surface $f$. Of course the collection $\left\{\phi_{m-3}\right\}$ forms likewise a $\mho^{p}$.

One-forms of the second kind. Same type of reduction to (3.2) $\bmod$ a $d \omega^{0}$, save that $\phi_{m-3}$ is replaced by some $\phi_{s}$. Their vector space $\bmod d K(f)$ is a $\mathcal{V}^{2 p}$. 
One-forms of the third kind. They have a finite number of logarithmic points with residues whose sum is zero.

Some special properties of one-forms of the first kind. Let

$$
\psi=\sum_{h=0}^{r} \alpha_{k} \psi_{h}(x, y)=0
$$

be a linear system of polynomials linearly independent $\bmod f$ and of common degree. Let the general $\psi$ intersect $f$ in a set of points $P_{1}, \cdots, P_{s}$ which includes all the variable points and perhaps some fixed points. The collection of all such sets is a linear series of degree $n$ and dimension $r$. The series is complete when its sets do not belong to an amplified series of the same degree: designation $g_{n}^{r}$ (concepts and terminology of Brill and Noether).

(3.4) TheOREM of ABel. Let $d u$ be any one-form of the first kind; let $\left\{P_{h}\right\}$ be any element of $a g_{n}^{r}$ and let $A$ be a fixed point of $f$. Then with integration along paths on $f$ :

$$
\sum \int_{A}^{P_{h}} d u=v
$$

is a constant independent of the element $\left\{P_{h}\right\}$ of $g_{n}^{r}$.

Still another classic, a sort of inverse of Abel's theorem is this:

(3.5) THEOREM OF JACOBI. Let $\left\{d u_{h}\right\}$ be a base for the one-forms of the first kind. Then for general values of the constants $v_{h}$ (exceptions noted) the system

$$
\sum_{k=1}^{p} \int_{A}^{P_{k}} d u_{h}=v_{h}
$$

in the $p$ unknowns $P_{k}, k \leqq p$, has a unique solution.

Periodic properties. Let $\left\{d u_{h}\right\}$ be as just stated and let $\left\{\gamma_{\mu}^{1}\right\}$, $\mu \leqq 2 p$ be an integral homology base (see (5.4)) for the module of one-cycles of $f$. The expression

$$
\pi_{h \mu}=\int_{\gamma_{\mu}^{1}} d u_{h}
$$

is the period of $\int d u_{h}$ as to the cycle $\gamma_{\mu}^{1}$. Let the matrix

$$
\Pi=\left[\pi_{h \mu}\right] ; \quad h, \mu \leqq 2 p ; \quad \pi_{h+p, \mu}=\tilde{\pi}_{h \mu}, h \leqq p .
$$

By means of integration on the Riemann surface $f$, Riemann has obtained the following comprehensive result (formulation of Scorza): 
(3.6) Theorem of Riemann. There exists an integral skew-symmetric $2 p \times 2 p$ matrix $M$ with invariant factors unity such that

$$
i \Pi M \Pi^{\prime}=\left[\begin{array}{ll}
0 & A \\
A^{*} & 0
\end{array}\right], \quad\left(A^{*}=\bar{A}^{\prime}\right)
$$

is a positive definite hermitian matrix.

Riemann matrices. This is the name given by Scorza to a matrix like $\Pi$ satisfying a relation (3.7) except that $M$ is merely rational skew-symmetric. The theory of such matrices has been extensively developed by Scorza [6]. He called $M$ : principal matrix of $\Pi$.

It may very well happen that there is more than one rational skewsymmetric matrix $M$ satisfying a relation (3.7) but without necessarily the positive definite property. These matrices are called singularity matrices. They form a rational vector space whose dimension $k$ is the singularity index of the Riemann matrix (Scorza).

\section{TOPOLOGY}

4. Results of Poincaré. Let $M^{n}$ be a compact orientable $n$-manifold which admits a cellular subdivision with $\alpha_{k} k$-cells (well-known property for varieties). The characteristic is the expression

$$
\chi\left(M^{n}\right)=\sum(-1)^{k} \alpha_{k} .
$$

The following two relations were proved by Poincaré:

$$
\begin{aligned}
\chi\left(M^{n}\right) & =\sum(-1)^{k} R_{k} \\
R_{k} & =R_{n-k}
\end{aligned}
$$

where $R_{k}$ is the $k$ th integral Betti number of $M^{n}$ : maximum number of linearly independent $k$-cycles with respect to homology (=with respect to bounding).

5. Intersections. In my work on algebraic geometry I freely used the intersection properties described below; they were actually justified and proved topologically invariant a couple of years later in my paper in the 1926 Transactions and much more fully in [10].

Let $M^{n}$ be as before and let $\gamma^{p}$ and $\gamma^{q}$ be integral $p$ - and $q$-cycles of $M^{n}$. One may define the intersection $\gamma^{p} \cdot \gamma^{q}$ and it is a $(p+q-n)$-cycle.

(5.1) If $\gamma^{p}$ or $\gamma^{q} \sim 0$ (bounds), then also $\gamma^{p} \cdot \gamma^{q} \sim 0$.

The more important situation arises when $p+q=n$. The intersection (geometric approximation) is then a zero-cycle

$$
C^{0}=\sum s_{j} A_{j}
$$


where the $s_{j}$ are integers. The intersection number

$$
\left(\gamma^{p}, \gamma^{n-p}\right)=\sum s_{j}
$$

is independent of the approximation. One proves readily

$$
\left(\gamma^{p}, \gamma^{n-p}\right)=(-1)^{(n-p) p}\left(\gamma^{n-p}, \gamma^{p}\right) .
$$

A basic result is:

(5.3) Theorem. $A$ n.a.s.c. in order that $\lambda \gamma^{p} \sim 0, \lambda \neq 0$ is that

$$
\left(\gamma^{p}, \gamma^{n-p}\right)=0
$$

for every $\gamma^{n-p}[9$, p. 15], [10, p. 78].

(5.4) Homology BASE. The collection $\left\{\gamma_{h}^{p}\right\}, h \leqq R_{p}$ is a homology base for the $p$-cycles when the $\gamma_{h}^{p}$ are independent and every $\gamma^{p}$ satisfies a relation

$$
\lambda \gamma^{p} \sim \sum s_{h} \gamma_{h}^{p}, \quad \lambda \neq 0 .
$$

(5.5) $A$ n.a.s.c. in order that the $\left\{\gamma_{h}^{p}\right\}, h \leqq R_{p}$ be a homology base for $p$-cycles is the existence of a set of $R_{p}$ cycles $\left\{\gamma_{k}^{n-p}\right\}$ such that the determinant

$$
\left|\left(\gamma_{h}^{p}, \gamma_{k}^{n-p}\right)\right| \neq 0 .
$$

Then $\left\{\gamma_{k}^{n-p}\right\}$ is likerwise a homology base for $(n-p)$-cycles.

6. The surface $F$. Orientation. Let $P$ be a point of $F$ and let $u=u^{\prime}+i u^{\prime \prime}, \quad v=v^{\prime}+i v^{\prime \prime}$ be local coordinates for $P$. Orient $F$ by naming the real coordinates in the order $u^{\prime}, u^{\prime \prime}, v^{\prime}, v^{\prime \prime}$. There results a unique and consistent orientation throughout the surface $F$. Hence $F$ is an orientable $M^{4}$.

Similarly if $C$ is a curve of $F$ and $u$ is a local coordinate at a nonsingular point $Q$ of $C$. The resulting orientation turns $C$ into a definite two-cycle, still written $C$.

Let $D$ be a second curve through $Q$, for which $Q$ is nonsingular and not a point of contact of the two curves. Then $Q$ contributes +1 to both the intersection number $(C, D)$ and to the number $[C D]$ of geometric intersections of $C$ and $D$. This holds also, through certain approximations when $Q$ is a multiple intersection. Hence always

$$
(C, D)=[C D]
$$

I will return to these questions later. 
7. Certain properties of the surface $F$. Its characteristic. To be a little precise let for a moment $F^{*}$ denote the nonsingular predecessor of $F$ in projective $S^{k+2}$. One may always choose a model $F^{*}$ of the function field $K(F)$ whose hyperplane sections are in general of a fixed genus $p>0$. We pass now to a cartesian representation of degree $m$ :

$$
F(x, y, z)=0
$$

which is a general projection of $F^{*}$ and in particular in general position relative to the axes. The general scheme that follows is due to Picard. Let $\left\{H_{v}\right\}$ be the pencil cut out by the planes $y=$ const., and let $a_{h}, h \leqq N$, be the values for which the planes $y=a_{h}$ are tangent to $F$. Then the following properties hold:

I. Every $H_{y}, y$ not an $a_{k}$, is of fixed genus $p$.

II. Every $H_{y}$ is irreducible.

III. The plane $y=a_{k}$ has a unique point of contact $A_{k}$ with $F$ and $A_{k}$ is a double point of $H_{a_{k}}$ with distinct tangents. Hence the genus of $H_{a_{k}}$ is $p-1$.

IV. Among the branch points of the function $z(x)$ taken on $H_{y}$ exactly two $\rightarrow A_{k}$ as $y \rightarrow a_{k}$.

V. The fixed points $P_{1}, \cdots, P_{m}$ of $H_{\nu}$ are all distinct.

I denote by $S_{y}$ the sphere of the complex variable $y$.

Characteristic. Cover $H_{y}$ with a cellular decomposition among whose vertices are the fixed points $P_{h}$ of the curve.

Then if $H_{y}^{*}=H_{y}-\sum P_{h}, \chi\left(H_{y}^{*}\right)=2-2 p-m$. Decompose also $S_{y}$ into cells with the $a_{k}$ as vertices. Were it not for these points, and since a sphere has characteristic two, $H_{y}^{*}$ promenading over $S_{\boldsymbol{y}}$ would generate a set $E=S_{y} \times H_{y}^{*}$ of characteristic

$$
\chi\left(E^{*}\right)=2(2-2 p-m) .
$$

Now in comparison with $H_{y}^{*}, H_{a_{k}}$ has lost two one-cycles, and has two points replaced by one. Hence

$$
\chi\left(H_{a_{k}}^{*}\right)=\chi\left(H_{y}^{*}\right)+1 .
$$

Upon remembering to add the missing points $P_{h}$ we have then

$$
\chi(F)=\chi\left(E^{*}\right)+N+m=(N-m-4 p)+4=I+4
$$

a formula due to J. W. Alexander (different proof). The number $I=N-m-4 p$ is the well-known invariant of Zeuthen-Segre. 
8. One-cycles of $F$. The first step was taken by Picard who proved this noteworthy result:

(8.1) TheOREM. Every one-cycle $\gamma^{1}$ of $F$ is $\sim$ a cycle $\gamma^{1}$ contained in an $H_{y}$.

The next important observation made by Picard was that $H_{v}$ contained a certain number $r$ of one-cycles which are invariant as $y$ varies. That is such a cycle $\gamma^{1}$ situated say in $H_{a}\left(a\right.$ not an $a_{k}$ ) has the property that as $y$ describes any closed path from $a$ to $a$ on the sphere $S_{y}$ the cycle $\gamma^{1}$ returns to a position $\gamma^{1} \sim \gamma^{1}$ in $H_{y}$. This draws attention to the nature of the variation $v \gamma^{1}$ of any cycle $\gamma^{1}$ under the same conditions.

Draw lacets $a a_{k}$ on $S_{y}$. Owing to $(7, I V)$ as $y$ describes $a a_{k}$ a certain cycle $\delta_{k}^{1}$ of $H_{\nu}$ tends to the point of contact $A_{k}$ of the plane $y=a_{k}$ and hence is $\sim 0$ on $H_{a_{k}}$. This is the vanishing cycle as $y \rightarrow a_{k}$. A simple lacet consideration shows that as $y$ turns once positively around $a_{k}$ the variation $\mathcal{V} \gamma^{1}$ of the cycle $\gamma^{1}$ is given by

$$
v_{\gamma}^{1}=\left(\gamma^{1}, \delta_{k}^{1}\right) \delta_{k}^{1}
$$

\section{Hence}

(8.3) Theorem. N.a.s.c. for invariance of the cycle $\gamma^{1}$ is that every

$$
\left(\gamma^{1}, \delta_{k}^{1}\right)=0
$$

A noteworthy generalization is obtained when $\gamma^{1}$ is replaced by a one-chain $L$ uniquely determined in term of $y$ provided that $y$ crosses no lacet. ${ }^{2}$ As $y$ turns as above around $a_{k}$ the variation of $L$ is

$$
\vartheta(L)=\left(L, \delta_{k}^{1}\right) \delta_{k}^{1}
$$

Noteworthy special cases are

I. $L$ is an oriented arc joining in $H_{y}$ two fixed points of $H_{y}$.

II. Let $C$ be an algebraic curve of $F$ and let $M_{1}, \cdots, M_{n}$ be its intersections with $H_{\nu}$. Then $L$ is a set of paths from a $P_{j}$ to every point $M_{k}$ in $H_{y}$.

(8.5) THEOREM. The number of invariant cycles of $H_{y}$ is equal to the Betti number $R_{1}(F)$ and both are even: $r=R_{1}=2 q$.

\footnotetext{
${ }^{2}$ In modern terminology, $L$ will be a relative cycle.
} 
This property was first proved in [7], although it was often admitted before. I give here an outline of the proof (not too different from the proof of [7]). ${ }^{3}$

To make the proof clearer I will use the following special notations: $\Gamma$ a 3-cycle of $F ;\left\{\Gamma_{h}\right\}$ base for the $\Gamma$ 's; $\gamma=\Gamma H_{y}$ : (one-cycle of $H_{y}$ ); $\left\{\alpha_{h}\right\}, h \leqq 2 p$, base for one-cycles of $H_{y}$;

$\left\{\beta_{j}\right\}, j \leqq 2 p-r$, base for the one cycles of $H_{y}$, none invariant; $\beta$ any linear combination of the $\beta_{j}$;

Matrices such as $\left[\left(\beta_{h}, \Gamma_{k}\right)\right]_{F}$ will be written $[\beta \Gamma]_{F}$.

Proof that $r=R_{\mathbf{1}} . \gamma=\Gamma H_{\nu}$ is invariant; conversely $\gamma$ invariant is a $\Gamma H_{y}$. Moreover $\gamma \sim 0$ in $H_{y}$ and $\Gamma \sim 0$ in $F$ are equivalent. Hence $\left\{\gamma_{h}\right\}$, $h \leqq R_{1}$, is a base for invariant cycles and therefore $r=R_{1}$.

Proof that $r$ is even. Since no $\beta$ is invariant, [ $\beta \delta]$ is of rank $2 p-r$. Hence there exist $2 p-r$ cycles $\delta$ which are independent in $H_{y}$. Denote them by $\bar{\delta}_{h}, h \leqq 2 p-r$. Since $\left(\gamma_{k} \delta_{h}\right)=0$ for every $k$, the $\delta_{h}$ depend on the $\beta_{j}$ in $H_{y}$. Hence one may take $\left\{\gamma_{h} ; \bar{\delta}_{k}\right\}$ as base for the one-cycles of $H_{y}$. Hence

$$
[\gamma \delta]=\left[\begin{array}{cc}
{[\gamma \gamma]} & 0 \\
0 & {[\delta \delta]}
\end{array}\right]
$$

is nonsingular. It follows that $[\gamma \gamma]$ is likewise nonsingular. Since it is skew-symmetric, a well-known theorem of algebra states that $r$ is even.

9. The two-cycles of $F$. From the expression (7.2) of the characteristic we have

$$
\chi(F)=I+4=R_{2}-2 R_{1}+2 .
$$

Hence

$$
R_{2}=I+2 R_{1}+2 .
$$

Besides this formula it is of interest to give an analysis of the 2-cycles.

Given a $\gamma^{2}$ one may assume it such that it meets every $H_{y}$ in at most a finite set of points. Let $Q$ be one of these and let $P, Q$ be a directed path from the fixed point $P$ to the point $Q$ in $H_{y}$. Call $L$ the sum of these paths. As $y$ describes $S_{\nu}-\sum$ lacets $a a_{k}, L$ generates a 3-chain $C^{3}$ whose boundary $\partial C^{3}$ consists of these chains:

(a) As $y$ describes $a a_{k}$ the vanishing one-cycle $\delta_{k}^{1}$ of $H_{y}$ generates a 2-chain $\Delta_{k}$ whose boundary

\footnotetext{
8 The point here is to prove that an invariant cycle, which is also a vanishing cycle, is necessarily zero.
} 


$$
\partial \Delta_{k}=\left(\delta_{k}^{1}\right)_{H_{a}} .
$$

The corresponding contribution to $\partial C^{3}$ is $\mu_{k} \Delta_{k}$, where (Zariski)

$$
\mu_{1}=\left(L, \delta_{1}^{1}\right), \quad \mu_{k}=\left(L+\mu_{1} \delta_{2}^{1}+\cdots+\mu_{k-1} \delta_{k-1}^{1}, \delta_{k}^{1}\right) .
$$

(b) A part $\left(H_{a}\right)$ of $H_{a}$.

(c) $-\gamma^{2}$ itself.

Hence

$$
\partial C^{3}=-\gamma^{2}+\sum \mu_{k} \Delta_{k}+\left(H_{a}\right) \sim 0
$$

and so

$$
\gamma^{2} \sim \sum \mu_{k} \Delta_{k}+\left(H_{a}\right)
$$

Since the right side is a cycle, and $y=a$ is arbitrary we have

$$
\sum \mu_{k} \delta_{k}^{1} \sim 0 \quad \text { in } H_{y}
$$

Conversely when (9.4) holds, (9.3) is a 2-cycle. Thus to obtain $R_{2}$ it is merely necessary to compute the number of linearly independent relations (9.4) and add to them one unit for all $\mu_{k}$ zero, that is for the cycle $H_{a}$ itself. This yields again (9.1).

For purposes of counting certain double integrals Picard required the number of finite 2-cycles independent relative to homologies in $F-H_{\infty}$. This is the number $R_{2}(F-H)$ and he found effectively

$$
R_{2}(F-H)=R_{2}-1 \text {. }
$$

10. Topology of algebraic varieties. I have dealt with it at length in both [8] and [9]. Questions of orientation and intersection are easily apprehended from the case of surfaces. I shall only recall here a few properties that are not immediate derivatives from the case of a surface.

The designations $V^{n}, H_{y}$ are the same as in Chapter I. The following properties are taken from [9, Chapter V]. The symbol $\boldsymbol{\gamma}^{k}$ will represent a $k$-cycle of $V^{n}$.

I. Every $\gamma^{k}, k<n-1$, of $H_{y}$ is invariant.

II. Every $\gamma^{k}, k<n$, of $V^{n}$ is $\sim \gamma^{k^{\prime}}$ in $H_{y}$.

III. When $k \leqq n-2, \gamma^{k} \sim 0$ in $V^{n}$ and $\gamma^{k^{\prime}} \sim 0$ in $H_{\nu}$ are equivalent relations.

IV. Under the same conditions $R_{k}\left(V^{n}\right)=R_{k}\left(H_{v}\right)$. 


\section{Analysis with Little Topology}

This is a rapid résumé of the extensive contributions of Picard, Severi and Poincaré upon which I applied topology (see IV). I will continue to consider the same surface $F$ and all notations of II.

11. Emile Picard and differentials on a surface. During the period 1882-1906 Picard developed almost single-handedly the foundations of this theory. His evident purpose was to extend the Abel-Riemann theory and this he accomplished in large measure. Reference: PicardSimart [2].

Picard studied particularly closed $\omega^{1}$, that is

$$
\omega^{1}=\alpha d x+\beta d y, \quad \partial \alpha / \partial y=\partial \beta / \partial x
$$

and $\omega^{2}$. The choice of closed $\omega^{1}$ is very appropriate since then $\int \omega^{1}$ is an element of $K(F)$, and analytic function theory plus topology are fairly readily available. ${ }^{4}$

For closed one-forms the same three kinds as for abelian differentials are distinguished, save that for the third kind logarithmic curves replace logarithmic points.

Significant results are

I. Closed one-forms of the first kind make up a $v^{q}$ (Castelnuovo) ( $q=\frac{1}{2} R_{1}$ as I have shown).

II. For the second kind same property save that they form a $v^{2 q}$ $\bmod d K(F)$. (Picard)

III. Regarding the third kind Picard obtained this noteworthy result: There exists a least number $\rho \geqq 1$ such that any set of $\rho+1$ curves are logarithmic for some closed $\omega^{1}$ having no other poles.

The 2-forms admit again three kinds: (a) first kind: holomorphic everywhere; (b) second kind: holomorphic to within a $d \omega^{1}$ about each point; (c) the rest. The third kind is characterized by the possession of periods: residues over some 2 -cycle $\boldsymbol{\gamma}^{2}$ bounding an arbitrarily small neighborhood of a one-cycle on a curve.

The 2 -forms of the first kind were already found by Max Noether. They are of the type

$$
\omega^{2}=\frac{Q(x, y, z) d x d y}{F_{z}^{\prime}}
$$

where $Q$ is an adjoint polynomial of degree $m-4$. These $\omega^{2}$ (or the associated $Q$ ) make up a $\vartheta^{p_{g}}$, where $p_{g}$ is the geometric genus of $F$, studied at length by Italian geometers.

${ }^{4}$ Strictly speaking, $\int \omega^{1}$ is in $K(F)$ only if $\omega^{1}$ has no residues or periods, but since $d \omega^{1}=0, \int \omega^{1}$ is invariant under a continuous variation in the path of integration. 
Let $\mho^{\rho_{0}}$ be the vector space of the $\omega^{2}$ of the second kind $\bmod d \omega^{1}$. Picard utilized his topological description of finite 2-cycles to arrive at the following formula:

$$
\rho_{0}=I+4 q-\rho+2 .
$$

12. Severi and the theory of the base. The central idea here is a notion of algebraic dependence between curves on the surface $F$. I must first describe this concept.

Let the nonsingular surface $F$ be in an $S^{k+2}$. A linear system of hypersurfaces of the space cuts out on $F$ a linear system of curves $|C|$. This system is complete if its curves are not curves of an amplified linear system.

We owe to the Italian School the following property: Every sufficiently ample complete system $|C|$ is part of a collection $\{C\}$ of $\infty^{q}$ such systems. The elements $|C|$ of the collection are in an algebraic one-one correspondence with the points of an abelian variety $V^{q}$, unique for $F$ and called sometimes the Picard variety of $F$ (see $\$ 18)$.

A system $\{C\}, \infty^{2}$ at least, without fixed points and with irreducible generic curve is said to be effective. Its curves are also called effective.

Note the following properties:

(a) An effective system is fully individualized by any one of its curves.

(b) The generic curves of an effective system have the same genus, written $[C]$.

(c) The curves $C, D$ of two effective systems intersect in a set of distinct points whose number is denoted by $[C D]$. In particular we write $\left[C^{2}\right]$ for $[C C]$ and $\left[C^{2}\right]$ is the degree of $C .^{5}$

(d) With $C, D$ as before let two curves $C, D$ taken together be individuals of an effective system $\{A\}$. This system is unique and we write

$$
A=B+C \text {. }
$$

(e) Any two curves $A_{1}, A_{2}$ of an effective system $\{A\}$ may be joined in $\{A\}$ by a continuous system $\infty^{1}$ of curves of $\{A\}$, whose genus, except for those of $A_{1}$ and $A_{2}$, is fixed and equal to [ $\left.A\right]$.

As an application of (e) let $A, B, C$ be effective and $A=B+C$. Following Enriques, connect $A$ to $B+C$ as indicated in (e). There follows a relation

- This degree should not be confused with the degree of $C$ as an algebraic curve in projective space. 


$$
\chi(A)+[B C]=\chi(B)+\chi(C)-[B C] .
$$

Hence if we define

$$
\phi(A)=\chi(A)+\left[A^{2}\right]=2-2[A]+\left[A^{2}\right],
$$

we verify at once that

$$
\phi(A)=\phi(B+C)=\phi(B)+\phi(C) .
$$

That is $\phi(A)$ is an additive function on effective systems.

When (12.1) holds between effective systems we set

$$
C=A-B
$$

and we have

$$
\phi(C)=\phi(A-B)=\phi(A)-\phi(B) .
$$

Note also that as regards the symbols $[B C]$ we may operate as with numbers, that is

$$
[(B \pm C) D]=[B D] \pm[C D] .
$$

Virtual systems: Let $\{A\},\{B\}$ be effective systems. Without imposing any further condition define a virtual system $\{C\}=\{A-B\}$ as the pair of symbols $\{\phi(A)-\phi(B)\},\left[(A-B)^{2}\right]$. This defines automatically $[C]$ and $\left[C^{2}\right]$. It is also clear that they are the same for $A-B$ and $A+D-(B+D)$ whatever $D$ effective. In other words $\{C\}$ depends only upon the difference $A-B$. The symbol $\{C\}$ is called a virtual algebraic system of curves and $[C],\left[C^{2}\right]$ are the related virtual genus and degree.

It may very well happen that while $A, B$ are effective there exist curves $C$, not necessarily effective such that $B+C$ ( $B$ together with $C$ ) is a member of $\{A\}$. If so $C$ is considered as a curve of the virtual system $\{C\}$ and has virtual characters $[C]$ and $\left[C^{2}\right]$, not necessarily its actual characters.

If we define $\{0\}=\{A-A\}$, as a virtual curve 0 is unique. One readily finds that $[0]=1,\left[0^{2}\right]=0$.

To sum up, the totality of effective and virtual curves form a module $M_{S}$ over the in tegers: the Severi module. Within $M_{s}$ a relation

$$
\lambda_{1} C_{1}+\cdots+\lambda_{8} C_{8}=0
$$

has a definite meaning. It is a relation of algebraic dependence between curves of $F$ in the sense of Severi.

The following remarkable result was proved by Severi: 
(12.3) Theorem of Severi. The module of curves of $F$ has a base consisting of $\rho$ effective curves $C_{1}, C_{2}, \cdots, C_{\rho}$, where $\rho$ is the Picard number relative to closed $\omega^{1}$ of the third kind.

That is any curve $C$ satisfies a relation

$$
\lambda C=\lambda_{1} C_{1}+\cdots+\lambda_{\rho} C_{\rho}
$$

where $\lambda$ and the $\lambda_{h}$ are integers and $\lambda \neq 0$.

Severi also proved

(12.4) The base may be chosen minimal, that is such that

$$
\lambda C=\lambda \sum \lambda_{h} C_{h}
$$

Moreover there exist effective curves $D_{1}, D_{2}, \cdots, D_{\sigma-1}$ such that actually

$$
C=\sum \lambda_{h} C_{h}+\sum \mu_{j} D_{j}
$$

One assumes, as one may that $\sigma$ is the least possible.

Severi also proved the following criteria:

(12.5) $A$ n.a.s.c. in order that the curves $C_{1}, C_{2}, \cdots, C_{8}$ be algebraically independent is that, with $H$ a plane section, the matrix

be of ranks.

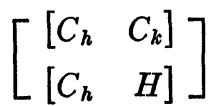

(12.6) N.a.s.c. in order that $\left\{C_{h}\right\}, h \leqq \rho$, be a base is that the determinant $\left|\left[C_{h} C_{k}\right]\right| \neq 0$ and that its order $\rho$ be the highest order for which this holds. Moreover, $\rho$ is the Picard number.

13. Poincaré and normal functions. Through an ingenious application of the theorems of Abel and Jacobi Poincaré arrived at a rapid derivation of some of the major results of Picard and the Italian geometers. I shall mainly deal with the part referring to Severi's theory of the base.

Let me first put in a most convenient form due to Picard and Castelnuovo the $\omega^{1}$ of the first kind of the curve $H_{\nu}$. A base for them may be chosen of type

$$
d u_{s}=\frac{Q_{s}(x, y, z) d x}{F_{z}^{\prime}}, \quad s \leqq p
$$

where $Q_{s}$ is an adjoint polynomial of degree $m-3$ in $x$ and $z$. For the first $p-q$ the polynomial is of degree $m-3$ in $x, y$ and $z$. For $s=p-q$ $+1, \cdots, p$, it is of degree $m-2$ in $x, y, z$. Actually within this last range one may choose the $Q_{h}$ so that the $d u_{h}$ only have constant pe- 
riods relative to the invariant cycles and zero relative to the rest. As for the first $p-q$ they will have zero periods relative to the invariant cycles.

Let $C$ be a curve on $F$ and $M_{1}, \cdots, M_{n}$ its intersections with $H_{y}$. The sums from a fixed point $P_{1}$ of $H_{y}$ to the $M_{k}$

$$
\sum_{k} \int_{P_{1}}^{M_{k}} d u_{s}=v_{s}, \quad s \leqq p,
$$

(integration in $H_{y}$ ) are Poincaré's normal functions.

Let $L$ be the set of integration paths and with $\delta_{k}^{1}$ as in (8) let

$$
\Omega_{k s}=\int_{\substack{\delta 1 \\ k}} d u_{s}
$$

Then with the $\mu_{k}$ as in (9.2) we find

$$
\begin{aligned}
v_{h} & =\sum \frac{\mu_{k}}{2 \pi i} \int_{a}^{a_{k}} \frac{\Omega_{k h}(Y) d Y}{Y-y} \quad h=1,2, \cdots, p-q \\
v_{p-q+j} & =\alpha_{j} \text { (constant) } \quad j=1,2, \cdots, q .
\end{aligned}
$$

REMARK. The only condition imposed upon the points $M_{k}$ is that they be rationally defined together on $H_{y}$. They may represent for example the following special cases: (a) any sum of multiples of the fixed points $P_{h}$ of $H_{y}$, in particular they may represent just $\mu P_{h}$; (b) if $C$ is reducible say $C=C_{1}+C_{2}$ with $M_{1 h}$ and $M_{2 h}$ as respective intersections one might have any set $t_{1} \sum M_{1 h}+t_{2} \sum M_{2 h}$, and similarly for several reducible curves; (c) any combination of the preceding two cases. In what follows, "curve" must be understood to include all these special cases.

As usual when dealing with abelian sums the $v_{\diamond}$ are only determined mod periods of the related $u_{\mathrm{s}}$.

(13.4) Theorem of Poincaré. N.a.s.c. to have a set of $v_{s}$ given by (13.3) represent a curve by means of Jacobi's inversion theorem are

$$
\sum_{h} \mu_{h} \Omega_{h s}(a)=0, \quad s=1,2, \cdots, p .
$$

(13.4b) Let $P(x, y, z)$ be any linear combination of the $P_{s}(x, y, z)$ divisible by $y-a$ and let

$$
d u=\frac{P(x, y, z) d x}{F_{z}^{\prime}}, \quad \Omega_{k}(y)=\int_{\substack{\delta 1 \\ b}} d u .
$$


Then one must have

$$
\sum \mu_{k} \int_{a}^{a_{k}} \Omega_{k}(y) d y=0 .
$$

(13.5) Comparison with Severi's results. Let the collection $\left\{\mu_{s}\right\}$ of the $\mu_{\varepsilon}$ occurring in any set of normal functions be designated by $\mu$. The collection $\{\mu\}$ is a module $U$. Let $U_{0}$ be the submodule of all the elements $\mu^{0}$ corresponding to the $\sum t_{h} P_{h}, t_{h}$ an integer. The quotient $U_{1}=U / U_{0}$ is the factor-module corresponding to all the curves which are not a plane section or more generally a $\sum t_{j} P_{j}$. The $U_{1}$ module has a base made up of $\rho-1$ algebraically independent curves and a minimum base consisting of $\rho+\sigma-2$ curves. By adding the $\mu(H)$ one has respectively $\rho$ and $\rho+\sigma-1$ for base and minimum base.

The quotient module $U_{1}=U / U_{0}$ is the module of all $\mu$ of curves none a plane section $H$. The module $U_{1}+H=M_{p}$ is the Poincare module and it is isomorphic with the Severi module $M_{8}$.

(13.6) REMARK. In order to get rapidly to the "heart of the matter" I have assumed at the outset that in (13.1) the polynomials $Q_{p \rightarrow q+j}$ were of degree $m-2$ in $x, y, z$. This was based upon rather deep results of Picard and Castelnuovo. Poincaré however merely assumed that the degree of $Q_{p-q+j}$ was $m-2+\nu_{j}$. As a consequence in (13.3) the constants $\alpha_{j}$ must be replaced by polynomials $\alpha_{j}(y)$ of degree $\nu_{j}$. Then Poincare shows that on the strength of the theorems of Abel and Jacobi every $\nu_{j}=0$ hence the $\alpha_{j}(y)$ must be constants and one has in fact the form (13.3).

Notice also that from the form of the $Q_{m-3+j}$ one may find another adjoint polynomial $R_{m-3+j}$ of degree $m-3$ in $y, z$ and $m-2$ in $x, y, z$ such that

$$
d w_{j}=\frac{Q_{m-3+j} d x+R_{m-3+j} d y}{F_{z}^{\prime}}
$$

is a closed $\omega^{1}$ of the first kind. The set $\left\{d w_{j}\right\}$ is then shown to be a base for such differentials. This proves rapidly that their "independent number" is $q$. Finally since the $\alpha_{j}$ are arbitrary constants the form of (13.3) shows implicitly that a complete (maximal) algebraic system of curves consists of $\infty^{q}$ linear systems in one-one correspondence with the points of an abelian $q$ dimensional variety (see IV, \$17).

In outline this shows how normal functions enabled Poincaré to obtain with ease a number of the major results of Picard and the Italian geometers. 


\section{Analysis with Topology}

14. On the Betti number $R_{1}$. In II I recalled my proof that $R_{1}$ is even and $R_{1}=2 q$, the number of invariant cycles of the curve $H_{y}$. This gave incidentally a direct topological proof that the number of independent one-cycles in any curve of a sufficiently general system was fixed and equal to $R_{1}$. It showed also that the irregularity $q$ of a surface, in the sense of Castelnuovo and Enriques was actually a topological character. As I will show in $\$ 17$, a topological proof that $q$ is an "absolute invariant" is immediate. Notice also that the distribution of complete algebraic systems in $\infty^{q}$ linear systems, referred to in (13.6) is also shown to have topological character.

(14.1) Let $\left\{d u_{k}\right\}, k \leqq q$, be a base for the closed $\omega^{1}$ of the first kind of $F$. On $H_{y}$ they coincide with the $u_{p-q+h}$ of $\$ 13$. Let $\pi_{k \mu}, \mu \leqq 2 q$, be the periods of $u_{k}$ relative to a homology base $\left\{\gamma_{\mu}^{1}\right\}, \mu \leqq 2 q$, for the one-cycles of $F$. From the fact that the periods of the differentials of the first kind of $H_{y}$ form a Riemann matrix, we infer:

(14.2) TheOREM. The matrix $\pi$ of the periods of the $u_{k}$ and their conjugates $\bar{u}_{k}$ as to the $\gamma_{\mu}^{2}$ is a Riemann $2 q \times 2 q$ matrix.

15. On algebraic two-cycles. A collection of mutually homologous 2-cycles is a homology class. In this manner algebraic cycles yield algebraic homology classes. Through addition they generate a module $M_{L}$. Thus in relation to the collection of curves on a surface $F$ there are three definite modules: $M_{S}$ (Severi module), $M_{P}$ (Poincaré module) and $M_{L}$ (Lefschetz module).

(15.1) Theorem. The three modules $M_{S}, M_{P}$ and $M_{L}$ are identical.

This property will be a final consequence of an extensive argument.

Returning to Poincaré's normal functions (III, §13) a glance at his two conditions for a set of normal functions to represent an algebraic curve reveals immediately that Poincaré's first condition simply means that

$$
\left\{\begin{array}{l}
\gamma^{2}=\sum_{\mu_{k} \Delta_{k}+}\left(H_{a}\right) \\
\sum \mu_{k} \delta_{k}^{1} \sim 0 \quad \text { in } H_{y}
\end{array}\right.
$$

is a cycle. As to the second condition it says merely that if

$$
\omega^{2}=\frac{Q(x, y, z) d x d y}{F_{z}^{\prime}}
$$


is of the first kind, that is if $Q$ is adjoint of order $m-4$, then

$$
\int_{\gamma 2} \omega^{2}=0
$$

Hence Poincaré's conditions are equivalent to the following result:

(15.3) TheOREM. $A$ n.a.s.c. for a cycle $\gamma^{2}$ to be algebraic is that the period of every 2-form of the first kind relative to $\gamma^{2}$ be zero.

(15.4) Remark. Among all the "algebraic" curves there were included all the sums $\sum m_{j} P_{j}$, where the $P_{j}$ are the fixed points of $H_{\boldsymbol{y}}$. It is evident that for these special "2-cycles" $\int \omega^{2}$ is zero.

(15.5) Corollary. Severi's number $\sigma$ is merely the order of the torsion group of the two-cycles (or equally of the torsion group of the onecycles).

For if $\gamma^{2}$ is a torsion 2-cycle we have $\lambda \gamma^{2} \sim 0, \quad \lambda \neq 0$, and hence

$$
\int_{\gamma 2} \omega^{2}=0
$$

for every $\omega^{2}$ of the first kind.

(15.6) Theorem. The number $\rho$ is the Betti number of algebraic cycles.

This is a consequence of the following property:

(15.7) Let $C_{1}, \cdots, C_{s}$ be a set of curves and let $\bar{C}_{h}$ be the cycle of $C_{h}$. Then

$\mathrm{P}_{\mathrm{a}}$ : algebraic independence of the $C_{h}$

$\mathrm{P}_{\mathrm{h}}$ : homology independence of the $\bar{C}_{\mathrm{h}}$ are equivalent properties.

From obvious considerations $P_{a}$ implies $P_{h}$. Conversely let $P_{h}$ hold. We must show that $\bar{C} \sim 0$ implies $\lambda C=0, \quad \lambda \neq 0$. Here I follow Albanese's rapid argument. Let $C=A-B, A$ and $B$ effective. Since $\bar{A} \sim \bar{B}$ and $[C D]=(\bar{C}, \bar{D})$ we have

$$
\left[A^{2}\right]=[A B]=\left[B^{2}\right] ; \quad[A H]=[B H]
$$

where $H$ is a plane section. Hence Severi's independence criterion is violated between $A$ and $B$. Consequently $\lambda A=\mu B, \quad \lambda \mu \neq 0$. From $[A H]=[B H]$ follows $\lambda=\mu$ and therefore $\lambda(A-B)=0=\lambda C, \quad \lambda \neq 0$. This proves (15.7).

It follows that $M_{S}=M_{L}$ and as $M_{P}=M_{S},(15.1)$ is proved.

Notice that we may now give the following very simple definition 
of virtual curve: it is merely an algebraic 2-cycle. Simplicity is even augmentable by replacing everywhere the symbol $=$ of algebraic dependence $(=)$ by the homology symbol $\sim$.

16. On 2-forms of the second kind. The basic result is the proof of the formula

$$
\rho_{0}=R_{2}-\rho \text {. }
$$

I shall just indicate an outline of my proof. I shall also show that the process outlined obtains incidentally Picard's fundamental result for $\rho$ concerning logarithmic curves of a closed $\omega^{1}$ of third kind. The steps follow closely an analogous outline in my monograph [9].

For convenience I call $\omega^{1}$ and $\omega^{2}$ regular when

$$
\omega^{1}=\frac{P d y-Q d x}{\phi(y) F_{z}^{\prime}}, \quad \omega^{2}=\frac{P d x d y}{\phi(y) F_{z}^{\prime}},
$$

where $P$ and $Q$ are adjoint polynomials and $\phi(y)$ is a polynomial.

If $\omega^{2}=d \omega^{1}, \omega_{2}$ is said to be improper. Thus $\rho_{0}$ is the dimension of the vector space of the $\omega^{2}$ of the second kind mod those which are improper.

By reduction of $\omega^{2}$ I understand the subtraction of an improper $\omega^{2}$.

I. The periods and residues of a normal 2-form are arbitrary.

II. One may reduce any $\omega^{2}$ of the second kind to the regular type.

III. A regular $\omega^{2}$ such that $\int \omega^{2}$ has neither residues nor periods is reducible to a regular $d \omega^{1}$.

Except for the presence of the polynomial $\phi(y)$ the proofs of the preceding propositions are very close to those of Picard. It is true that allowing $\phi(y)$ in regular $\omega^{1}$ and $\omega^{2}$ considerably simplifies every step (see $[\mathbf{9}$, Note I]).

IV. Let $C$ be a curve of order $s$. We may choose coordinates such that $C$ does not pass through any of the fixed points $P_{j}$ of $H_{y}$, nor through the points of contact of the planes $y=a_{k}$. One may form an $\omega^{1}=R d x$, $R \in K(F)$ possessing on $H_{y}$ the s-logarithmic points of $\mathrm{CH}_{y}$ with logarithmic period $2 \pi i$ and say $P_{1}$ with logarithmic period $-2 \pi i s$. One may even select $R$ so that $(\partial R / \partial y) d x$ has no periods. From this follows that there is an $S(x, y, z) \in K(F)$ such that

$$
\omega^{2}=d(R d x+S d y)
$$

is regular.

Take now $C_{1}, C_{2}, \cdots, C_{t}$ and the axes so chosen that they all behave like $C$. Let $\omega_{h}^{2}$ be analagous to $\omega^{2}$ for $C_{h}$. 
Owing to III it is now readily shown that n.a.s.c. in order that some linear combination

$$
\omega^{2}=\sum \alpha_{h} \omega_{h}^{1}
$$

be without periods is that the $C_{s}$ and $H$ be logarithmic curves of a closed $\omega^{1}$.

Since $R_{\mathbf{2}}$ is finite there is a least $\rho-1$ such that for $s=\rho$ the curve $C_{h}, H$ are logarithmic curves of a closed $\omega^{1}$ of the third kind. Hence

(16.2) Picard's fundamental property for $\rho$ is a consequence of the finiteness of the Betti number $R_{2}$.

V. To proceed one may form $\rho-1$ linearly independent $\omega^{2}$ which are improper. Since the total number of distinct periods is equal to $R_{2}(F-H)$ $=R_{2}-1$ we have then $\rho_{0}=R_{2}-\rho$, as asserted.

(16.3) On Picard's treatment of $\rho_{0}$ and $\rho$. Owing to lack of topological technique Picard proved directly that $\rho_{0}$ was finite by showing through strong algebraic operations that if

$$
\omega^{2}=\frac{Q(x, y, z) d x d y}{F_{z}^{\prime}}
$$

where $Q$ is adjoint, is of the second kind, the degree of $Q$ was bounded.

Although Picard did not observe it, his later treatment of $\omega^{2}$ of the second kind contained implicitly (argument of 16.2) the proof that $\rho$ had the property by which he defined it relative to closed $\omega^{1}$ of the third kind.

17. Absolute and relative birational invariance. Take again a general $n$-variety

$$
F\left(x_{1}, \cdots, x_{n}, y\right)=0 .
$$

Let $\left\{\xi_{0}, \cdots, \xi_{r}\right\}$ be a homogeneous base for the function field $K(F)$. Then the system

$$
\tau y_{h}=\xi_{h}, \quad h=0,1,2, \cdots, r>n
$$

represents a model $F_{1}$ of $F$ in the projective space $S^{r}$, with homogeneous coordinates $y_{h}$. If $\left\{\eta_{0}, \cdots, \eta_{s}\right\}, s>n$, is a second homogeneous base for $K(F)$, the system

$$
\sigma z_{k}=\eta_{k}, \quad k=0,1, \cdots, s
$$

represents a second model $F_{2}$ of $F$ in a projective space $S^{s}$. Since $\left\{\xi_{h}\right\}$ and $\left\{\eta_{k}\right\}$ are homogeneous bases for $K(F) F_{1}$ and $F_{2}$ are birationally transformable into one another. The simple example of two 
elliptic curves of degrees 3 and 4 show however that the corresponding structures need not be homeomorphic. The difficulty is caused by the presence of singularities. A standard device for curves enables one to "forget" singularities and restore homeomorphism. No such device is known for a $V^{n}, \quad n>1$.

For simplicity let me limit the argument to surfaces. I have really considered a surface as a nonsingular model in some projective space. Let $F_{1}, F_{2}$ be two such distinct models and suppose that the field $K(F)$ is not that of a ruled surface. Then according to Castelnuovo and Enriques a birational transformation $T: F_{1} \rightarrow F_{2}$ may take a finite number $\delta_{12}$ of exceptional points of $F$ into disjoint nonsingular rational curves. There exists an analogous $\delta_{21}$ for $T^{-1}$. Let a point $P$ of $F_{1}$ be sent by $T$ into a curve $C$ of $F_{2}$. Since $C$ is rational and nonsingular it is topologically a sphere. Hence its characteristic $\chi(C)=2$. Hence the gain in $\chi\left(F_{1}\right)$ through $\delta_{12}$ exceptional points is $\delta_{12}$. Therefore

$$
\chi\left(F_{1}\right)+\delta_{12}=\chi\left(F_{2}\right)+\delta_{21} .
$$

Now a character, numerical or other of $F$ is said to be an absolute invariant if it is unchanged under all transformations such as $T$. A relative invariant is one that may change under certain transformations $T$.

Let me examine some of the characters that have been introduced.

It is readily shown that under $T$ both $R_{2}$ and $\rho$ are increased by the same amount $\delta_{12}-\delta_{21}$. Hence both are relative invariants and $\rho_{0}=R_{2}-\rho$ is an absolute invariant.

Since

$$
\chi(F)=R_{2}-2 R_{1}+2
$$

and both $\chi$ and $R_{\mathbf{2}}$ vary in the same way, $\chi$ is a relative invariant and $R_{1}$ is an absolute invariant.

Therefore:

(17.3) The dimensions of the spaces of closed $\omega^{1}$ of the first and second kinds and of $\omega^{2}$ of the second kind are absolute invariants.

18. Application to abelian varieties. Let $\Pi$ and $M$ be a Riemann matrix and its principal matrix (see \$3).

Introduce the following vectors:

$$
\begin{gathered}
u=\left(u_{1}, \cdots, u_{2 p}\right), \quad u_{p+j}=\bar{u}_{j} \\
\pi_{\mu}=\left(\pi_{1 \mu}, \cdots, \pi_{2 p, \mu}\right), \quad \mu=1,2, \cdots, 2 p ; \quad \pi_{p+j, \mu}=\bar{\pi}_{j \mu} .
\end{gathered}
$$

Through the hyperplanes 


$$
u=\sum s_{\mu} \pi_{\mu},
$$

$s_{\mu}$ an integer, real $2 p$-space is partitioned in a familiar way into paralellotopes. A suitable fundamental domain $D$ is

$$
u=\sum t_{\mu} \pi_{\mu}, \quad 0 \leqq t_{\mu}<1 .
$$

The identification of congruent boundary points turns this domain into a $2 p$-ring $R^{2 p}$ (product of $2 p$ circles):

Corresponding to $I I$ and $M$ there may be defined a whole family of functions $\theta$ of various orders. Each such function $\phi$ is a holomorphic function in the domain $D$. Those of a given order, say $n$, are characterized by the property that $\phi_{n}\left(u+\pi_{\mu}\right)=\phi_{n}(u)$ times a fixed linear exponential function of $u$. I have shown that one may find an $n$ such that if $\left\{\theta_{n}^{j}(u+\alpha)\right\}, \alpha$ a fixed $p$-vector, $j=0,1, \cdots, r$, is a finite linear base for the $\theta_{n}(u+\alpha)$ then the system

$$
k x_{j}=\theta_{n}^{j}(u+\alpha)
$$

represents a nonsingular $p$-variety $V^{p}$ in projective $S^{r}$, and this $V^{p}$ is in analytic homeomorphism with the ring $R^{2 p}$. This is an abelian $p$-variety (see [8])

The topological relation $V^{p} \leftrightarrow R^{2 p}$ assigns an exceptionally simple topology to $V^{p}$. Let the edges of $D$ oriented from the origin out be designated by $1,2, \cdots, 2 p$. Any $i_{h}$ defines a one-cycle represented by $\left(i_{h}\right)$; any two edges $i_{h}, i_{k}$ define a 2 -cycle represented by $\left(i_{h}, i_{k}\right)$, etc. The $\left(i_{h}, i_{k}\right), i_{h}<i_{k}$ form a base for the 2-cycles of $V^{p}$, etc.

I am mainly concerned with the 2 -cycles. In view of $(\nu, \mu)=-(\mu, \nu)$ a general 2-cycle is represented by a homology

$$
\gamma^{2} \sim \sum m_{\mu \nu}(\mu, \nu), \quad m_{\mu \nu}=-m_{\nu \mu} .
$$

On the other hand

$$
\stackrel{2}{\omega_{j k}}=d u_{j} d u_{k} ; \quad j, k \leqq p ; \quad j<k
$$

is a closed 2-form of the first kind of $V^{p}$ and $\left\{d u_{h} d u_{k}\right\}$ is a base for all such forms.

(18.2) Remarks. On a general $n$-variety $V^{n}, n \geqq 2$. Considerations of the same type as in $\$ 12$ may be extended automatically to algebraic dependence of hypersurfaces of $V^{n}$ (its $V^{n-1}$ ), and also to their $(2 n-2)$-cycles. Algebraic and homology dependence give rise to a number $\rho\left(V^{n}\right)$. I single out especially the following proposition from [9, p. 104] (Corollary): 
(18.3) THEOREM. Let $\Phi$ be a fixed surface of $V^{n}$ (general intersection of hyperplane sections of $V^{n}$ ) and let $C_{1}, \cdots, C_{8}$ be hypersurfaces which cut $\Phi$ in curves $C_{h}^{*}, h \leqq s$. Then the following relations are all equivalent: relations of algebraic dependence between the $C_{h}$ in $V^{n}$, the same between the $C_{n}^{*}$ in $\Phi$; relations of homology between the standard oriented cycles $\bar{C}_{h}, C_{h}$ in $V^{n}$; the same for the $C_{h}^{*}$ in $\Phi$.

Returning now to the abelian variety $V^{p}$ let $\Phi, C, C^{*}$ be this time the same as above but for $V^{p}$. Now the $\omega_{h \mathrm{~h}}^{2}$ taken on $\Phi$ become $\omega^{2}$ of the first kind for $\Phi$. If $\left\{C_{s}\right\}, s \leqq \rho\left(V^{p}\right)$, is a base as to $=$, or equivalently as to $\sim$ and algebraic $(2 p-2)$-cycles of $V^{p}$, then the same holds for the curves $C_{s}^{*}$ in $\Phi$. Hence by theorem (15.2):

$$
\int_{c_{\imath}^{*}} d u_{j} d u_{l}=0 ; \quad j, l \leqq p
$$

On the other hand since the $(\mu, \nu)$ are cycles in $\Phi$ we have in $\Phi$

$$
C_{s}^{*} \sim \sum m_{\mu \nu}^{*}(\mu, \nu), \quad m_{\mu \nu}=-m_{\nu \mu} .
$$

Hence (18.4) yields

$$
\sum m_{\mu \nu}^{s} \pi_{j \mu} \pi_{k \nu}=0 ; \quad j, k \leqq p .
$$

This really means that the $\rho$ matrices $\left[m_{\mu \nu}^{s}\right]$ are linearly independent singularity matrices for the Riemann matrix $\Pi$. If the singularity index of $\Pi$ is $k$, then one must have

$$
\rho \leqq k .
$$

The possible inequality is due to the fact that an algebraic 2-cycle of $\Phi$ must satisfy a relation such as (18.4) not merely with respect to the closed $\omega^{2}$ of the first kind of $V^{p}$ in $\Phi$, but also with respect to all $\omega^{2}$ of the first kind of $\Phi$, and one cannot exclude the possible existence of such $\omega^{2}$ other than the closed taken on $\Phi$. However, the following two properties hold:

(a) There is a base for the forms $M$ made up of principal forms.

(b) Each principal $M$ gives rise to a particular system of functions $\phi$ à la $\theta$. These functions are said to be intermediary.

(c) If $\left\{M_{j}\right\}, j \leqq k$, is a base for the matrices $M$, and $\phi_{j}$ is an intermediary function relative to $M_{j}$ then $\phi_{j}=0$ represents a hypersurface of $V^{p}$ and these hypersurfaces are algebraically independent.

It follows that $\rho \geqq k$ and therefore

$$
\rho=k \text {. }
$$


This is the result that I was looking for.

Actually the relations between the hypersurfaces as cycles and their Severi independence are the same as for their sections with the surface $\Phi$. That is,

(18.8) ThEOREM. For hypersurfaces of $V^{p}$ algebraic dependence and homology in $V^{p}$ are equivalent relations.

\section{BibLIOGRAPHY}

1. Emile Picard, Triaté d'analyse. Vol. 2, Gauthier-Villars, Paris.

2. Emile Picard and George Simart, Théorie des fonctions algébriques de deux variables indépendantes. Vols. I, II, Gauthier-Villars, Paris, 1895, 1906.

3. Francisco Severi, Sulla totalità delle curve algebriche traceiate sopra una superficie algebrica, Math. Ann. 62 (1906), 194-226.

4. - La base minima pour la totalité des courbes tracées sur une surface algêbrique, Ann. Sci. Ecole Norm. Sup. 25 (1908), 449-468.

5. Henri Poincaré, Sur les courbes tracées sur une surface algébrique, Ann. Sci. Ecole Norm. Sup. 27 (1910), 55-108.

6. Gaetano Scorza, Intorno alla teoria generale delle matrici di Riemann e ad alcune sus applicaciones, Rend. Circ. Mat. Palermo 41 (1916), 263-380.

7. Solomon Lefschetz, Algebraic surfaces, their cycles and integrals, Ann. of Math. 21 (1920), 225-258.

8. - On certain numerical invariants of algebraic varieties with application to abelian varieties, Trans. Amer. Math. Soc. 22 (1921), 327-482.

9. - L'analysis situs et la géométrie algébrique, Borel Series, 1924.

10. - Topology, Amer, Math. Soc. Colloq. Publ., Vol. 12, Amer. Math. Soc., Providence, R. I., 1930; reprint Chelsea, New York, 2nd ed., 1950.

11. Oscar Zariski, Algebraic surfaces, Ergebnisse der Math., Springer-Verlag, Berlin, 1935; reprint Chelsea, New York, 1948.

12. Heisuki Hironaka, Resolution of singularities of an algebraic variety over a field of characteristic zero, Ann. of Math. (2) 79 (1964), 109-329.

13. W. V. D. Hodge, Theory and application of harmonic integrals, Cambridge Univ. Press, New York, 1941. 